\title{
Fabricating Elastomeric Photomask with Nanosized-Metal Patterns for Near-Field Contact Printing
}

\author{
Sangyoon Paik ${ }^{1,2,3 \dagger}$, Gwangmook Kim ${ }^{1,2 \dagger}$, Dongchul Seo ${ }^{1,2}$, and Wooyoung Shim ${ }^{1,2, *}$ \\ ${ }^{1}$ Department of Materials Science and Engineering, Yonsei University, Seoul 03722, Republic of Korea \\ ${ }^{2}$ Center for Multi-Dimensional Materials, Yonsei University, Seoul 03722, Republic of Korea \\ ${ }^{3}$ LCD TV Panel design team 2, LG Display Co., Ltd. Gyeonggi 10845, Republic of Korea
}

\begin{abstract}
When an elastomeric photomask is used for near-field contact printing, the high deformability of the elastomer mask plate enables gap-free full contact with the substrate, minimizing the effect of diffraction. This image-transfer technique provides sub-50 $\mathrm{nm}$ resolution and depth-of-focus-free lithographic capability with cost-efficient equipment. However, the method's application is limited due to the lack of a wellestablished protocol for fabricating a nanoscale mask pattern on an elastomeric substrate, which remains a major technical challenge in the field of near-field contact printing. In this study, we present a reliable protocol for fabricating a metal-embedded polydimethylsiloxane (PDMS) photomask. Our fabrication protocol uses conventional nanofabrication processes to fabricate nanosized chromium mask patterns and then transfers the chromium patterns to an elastomeric mask plate using a sacrificial Ni layer. Our protocol provides a high flexibility mask pattern design, and highly stable metal patterns during transferring process. By careful optimizing the experimental parameters, we determined a perfect pattern transfer ratio, which avoided any mechanical failure of the metal pattern, such as debonding or wrinkling. We then fabricated a PDMS photomask and confirmed its nanoscale patterning resolution, with the smallest feature $51 \mathrm{~nm}$ in width under a 400-nm light source. We anticipate that our fabrication protocol will enable the application of cost-efficient and high-resolution near-field photolithography.
\end{abstract}

(Received September 23, 2020; Accepted December 13, 2020)

Keywords: near-field photolithography, elastomeric photomask, transferring process

\section{Introduction}

The image-transfer capability of photolithography has improved significantly with the development of shadow printing and projection printing [1]. These two image-transfer techniques have complementary strengths and weaknesses. Shadow printing with photomasks in physical contact or in close proximity to photoresist surfaces is called contact printing and proximity printing, respectively. The methods can be simply implemented; however, there is a trade-off between the patterning resolution and mechanical stability of the mask depending on the distance between the mask and substrate. In projection printing, the mask is separated by

- 백상윤: 박사과정, 김광묵·서동철: 석박사통합과정, 심우영: 부교수 *Corresponding Author: Wooyoung Shim

[Tel: +82-2-2123-2857, E-mail: wshim@yonsei.ac.kr]

Copyright (C) The Korean Institute of Metals and Materials

'These authors contributed equally: Sangyoon Paik, Gwangmook Kim. many centimeters from the substrate, and lens elements are used to focus the mask pattern onto the substrate to provide high resolution. This technique offers advantages including better resolution, registration, and throughput, and thus replaces contact and proximity printing to fabricate leadingedge devices. However, it suffers from manufacturing difficulties, requires a sophisticated optical element to focus high-resolution images onto the substrate, which impacts cost efficiency.

Near-field contact printing using an elastomeric photomask is an alternative image-transfer technique that provides the high resolution of conventional contact printing, the low damage of proximity printing, and simple implementation and cost-effectiveness, compared to projection printing [2,3]. It takes advantage of an elastomeric mask plate that can deform itself to conform to the non-linear profile of a surface, providing full contact between the photoresist surface and the 


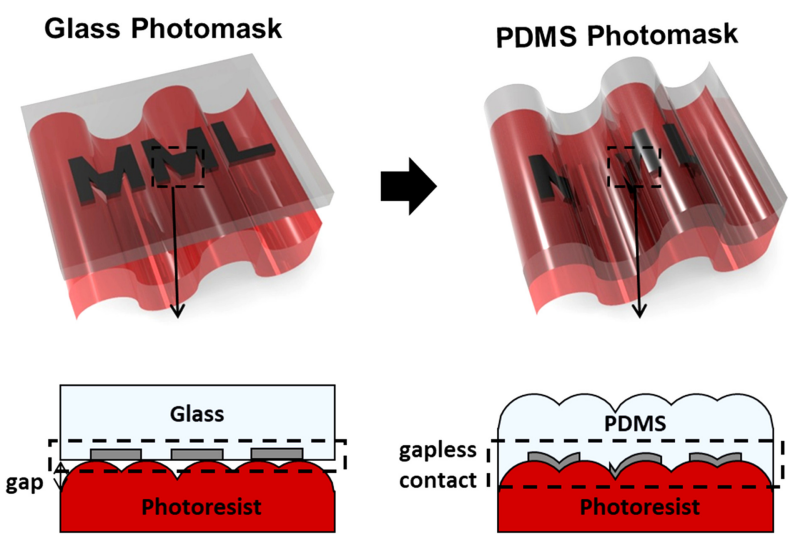

Fig. 1. Schematic illustration of contact printing onto a non-planar photoresist layer with a conventional glass photomask and an elastomeric photomask.

substrate, without a gap. In contrast to conventional contact printing, which entails the diffraction of light through a gap between a rigid photomask and a substrate [1], an elastomeric photomask suppresses the diffraction effect by conformal contact. This enables high resolution without any focusing element or short-wavelength light source (Figure 1) [2]. In addition, previous research has found that an elastomeric photomask can ensure the stability of metal patterns with engineered mechanical properties and geometric factors of the elastomeric mask plate and metal pattern [2]. This provides the advantages of the elastomeric photomask's flexible form factor [2,4-5], while providing depth-of-focusfree patterning of highly dense features on a curvilinear substrate, which could be useful for fabricating wearable and flexible devices [6-12].

A major technical challenge to near-field contact printing is the lack of a reliable protocol for fabricating elastomeric photomasks with nanoscale metal patterns. An elastomeric substrate such as polydimethylsiloxane (PDMS) is considered incompatible with conventional lithographic processes, because of its low surface energy $[13,14]$, which can result in poor adhesion between the photoresist and deposited metal. In addition, when the metal pattern is directly deposited on an elastomeric substrate, the mismatch in the thermal expansion coefficient (CTE) of the elastomeric substrate and metal film may cause the mechanical failure of the metal film $[15,16]$.

In attempts to avoid these issues, several studies have reported a method of mechanical transfer from a conventional silicon wafer to an elastomeric substrate that involves controlling the adhesion of the metal features [4,5]. However, at the nanoscale, the limited contact area of the metal patterns prevents stable bonding to the elastomeric substrate, despite adhesion engineering. Therefore, application of these approaches has been limited to micrometer-scale patterns, and the metal patterns were prone to mechanical failure during peeling off from the substrate. As an alternative, we have introduced a technique that uses metal patterns embedded in an elastomer photomask, which enables the fabrication of sub-diffraction patterns on a variety of non-flat surfaces [2].

In the present study, we demonstrate a reliable fabrication protocol for preparing a metal pattern-embedded-elastomeric photomask, where the metal pattern is not limited in shape or size. The proposed fabrication protocol involves the transfer of metal features from a silicon substrate to an elastomeric substrate using a sacrificial layer. This transfer method prevents the debonding of the pattern and minimizes mechanical stress during the transfer process. Using the proposed protocol, we fabricated an elastomeric photomask with nanosized-metal patterns, and demonstrated near-field contact printing capable of patterning features as small as $51 \mathrm{~nm}$. The proposed protocol provides a simple and reliable method for near-field photolithography using an elastomeric photomask, which facilitates the high-resolution imagetransfer process.

\section{Process Development}

Figure 2(a) shows a schematic illustration of the fabrication of a metal-embedded elastomeric photomask. The fabrication process consists of two main steps: (1) metal patterning on a silicon wafer and (2) embedding metal features in the PDMS mask plate. Our strategy exploits a well-established nanofabrication process for conventional substrates to define the metal mask pattern, and then transfer the metal features to an elastomeric substrate using a sacrificial layer. The advantage of this strategy is that it provides high flexibility in the design of the mask pattern, as with a conventional glass photomask, while preserving the precise dimensions of the mask patterns by preventing mechanical stress during the transfer process. Throughout the fabrication process, careful control of the details of experimental conditions is important to ensure the stability of the metal patterns. In this section, we 
(a)

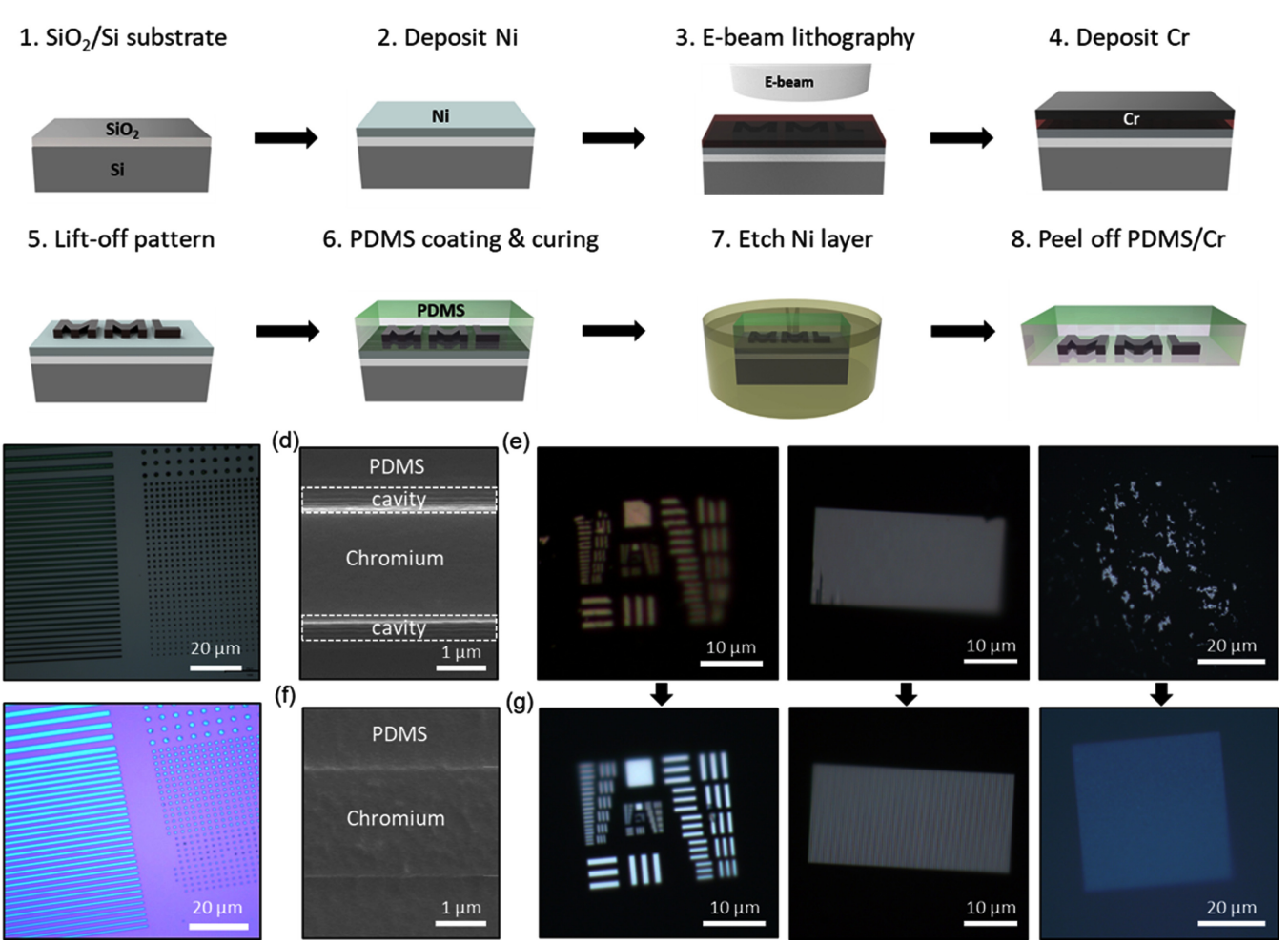

Fig. 2. (a) Fabrication process of PDMS photomask with nanosized chromium patterns. Optical microscopy images of (b) e-beam lithography patterns and (c) chromium patterns after the lift-off. (d) Scanning electron microscopy image of a chromium pattern embedded in PDMS mask plate without room-temperature aging. (e) Optical microscopy images of chromium pattern on PDMS mask plate without room-temperature aging. (f) Scanning electron microscopy image of a chromium pattern embedded in PDMS mask plate with roomtemperature aging. (g) Optical microscopy images of chromium pattern on PDMS mask plate with room-temperature aging.

will discuss the effect of experimental parameters in the fabrication process.

To fabricate mask patterns on a silicon wafer, we first cleaned a silicon wafer with a $3,000 \AA$ oxide layer by sonication with acetone and isopropyl alcohol for $10 \mathrm{~min}$ each. Then, a $200 \mathrm{~nm}$ nickel layer was deposited onto the silicon wafer as the sacrificial layer using a DC magnetron sputtering system. Notably, since all of the nanofabrication processes, such as e-beam lithography and metal deposition, will be performed on the nickel layer, the adhesion between a nickel layer and a silicon wafer needs to be stable enough to endure the temperature and chemical conditions during the process. Thermal stress in the nickel layer during the deposition process can degrade its adhesion to the silicon wafer, and may result in the delamination of the nickel layer [17]. To prevent thermal stress, we avoided sudden temperature changes during the deposition and used a sputtering process instead of an evaporation process.
After the deposition of the nickel sacrificial layer, we defined a mask pattern on the nickel layer using e-beam lithography (Fig. 2(b)). We used a bilayer of polymethyl methacrylate (PMMA) resist with different molecular weights to facilitate the lift-off process. After the development of the e-beam resist pattern, we deposited a 30 $\mathrm{nm}$ thick chromium layer by e-beam evaporation followed by lift-off with acetone (Fig. 2(c)). Regarding the design of the pattern, the ratio between the thickness and width of the chromium pattern should be considered to prevent the failure of the chromium pattern when the elastomeric photomask is deformed [2]. Higher thickness and smaller width results in a more stable chromium pattern. Usually, for a pattern several micrometers wide, a 30-nm thick chromium pattern is fine. If the width of the pattern becomes larger than tens of micrometers in width, the thickness of the chromium pattern should be increased.

The next step was to transfer a predefined chromium 
pattern onto a PDMS substrate. The surface of the substrate was cleaned to remove foreign particles before casting the PDMS onto the nickel sacrificial layer with chromium patterns. We experimentally determined that cleaning the substrate with solvents such as acetone and methanol could damage the chromium pattern on the nickel layer (e.g., partial debonding, cracking); therefore, PDMS was used to clean the substrate. In detail, PDMS with a 10:1 base/curing agent ratio was cast on the surface, followed by curing at $80^{\circ} \mathrm{C}$ for $4 \mathrm{~h}$. During solidification, the PDMS slab mechanically captured foreign particles on the surface. After cooling to room temperature, the PDMS slab was gently peeled off from the surface. Since adhesion between the PDMS and the chromium layer was weaker than the adhesion between the chromium and nickel layers, the PDMS slab can trap foreign particles on the surface but does not cause damage to the chromium patterns.

The cleaned substrate was exposed to $\mathrm{O} 2$ plasma (30 Torr, $100 \mathrm{~W}$ ) to improve adhesion between the chromium patterns and PDMS. Once the surface was activated by $\mathrm{O}_{2}$ plasma,
PDMS was cast onto the nickel sacrificial layer, followed by curing at $80^{\circ} \mathrm{C}$ for $4 \mathrm{~h}$. To ensure that the chromium patterns get perfectly covered with PDMS, the liquid PDMS on the substrate should be left at room temperature, around $20{ }^{\circ} \mathrm{C}$, for $48 \mathrm{~h}$ before the high-temperature curing. Because of the nanoscale dimensions of the chromium pattern, the area of the interface between the pattern and PDMS is extremely small, and therefore the contact area on the side of chromium is important to ensure the chromium pattern bonds to the PDMS mask plate. Curing PDMS without room-temperature aging can cause cavities on the sides of a chromium pattern (Fig. 2(d)), leading to a partial debonding of the pattern from the PDMS mask plate due to insufficient adhesion (Fig. 2(e)). In contrast, with sufficient aging, the PDMS mask plate thoroughly covers the chromium patterns, leaving no cavities (Fig. 2(f)), and the mask patterns were well transferred to the PDMS mask plate (Fig. 2(g)).

In the PDMS casting process the base/curing agent ratio of PDMS is an important consideration. In the structure of stiff islands on a complaint substrate such as elastomeric (a)

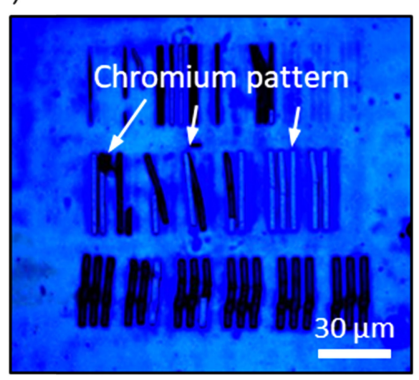

Base:curing agent $=30: 1$

(d)

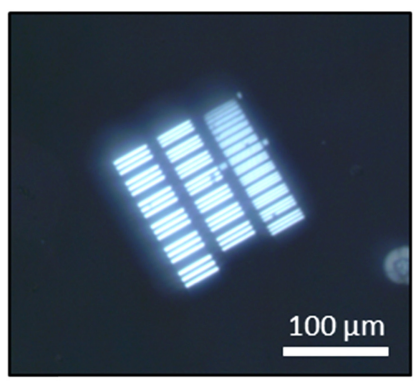

Base:curing agent $=30: 1$ (b)

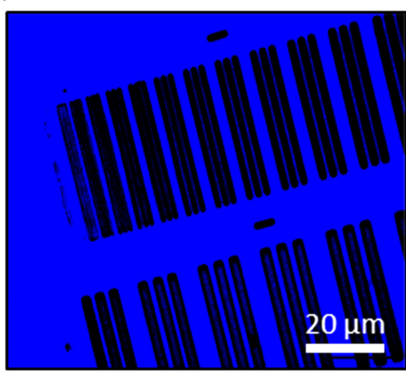

Base:curing agent $=8: 1$

(e)

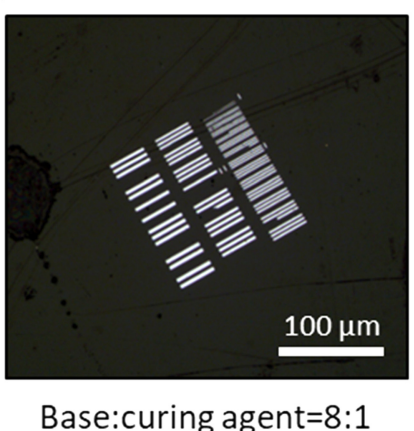

(c)

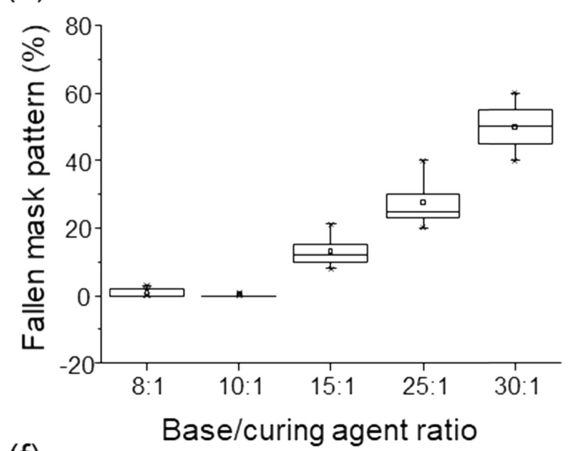

(f)

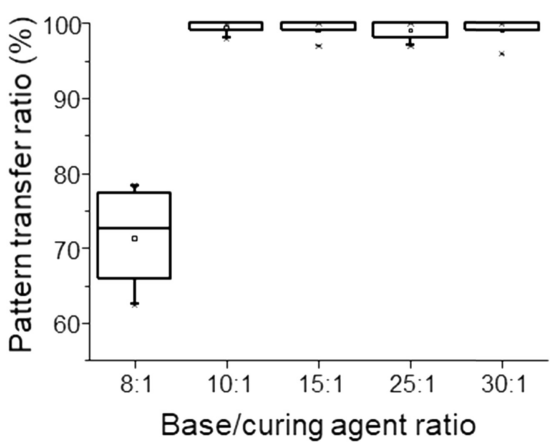

Fig. 3. Optical microscopy images of a patterned substrate using PDMS photomask with a different mixing ratio of base/curing agent, (a) 30:1 and (b) 8:1. (c) The ratio of a fallen mask pattern during photolithography depending on the mixing ratio of PDMS. Optical microscopy images of PDMS photomask after photolithography with a different mixing ratio of base/curing agent, (d) 30:1 and (e) 8:1. (f) The ratio of a pattern transfer from a silicon substrate to PDMS mask plate depending on the mixing ratio of PDMS. 
photomask, the mechanical properties, and geometry of the components determine the stability of the stiff islands (in our case, the chromium pattern) [18]. The mechanical properties of the PDMS mask plate are largely dependent on the base/ curing agent ratio [19]. If the mixing ratio of PDMS is not properly engineered, the unstable chromium pattern is easily debonded during the transfer process and lithography process. If the mixing ratio is too high, PDMS photomask is too soft and can easily stretch when the PDMS photomask partially sticks to the photoresist layer. This phenomenon induces tensile strain in the elastomeric photomask and then causes debonding of the chromium patterns after the lithography process (Figure 3(a)-(c)). However, if the ratio is small (8:1), the adhesion between the chromium patterns and PDMS is too weak, causing debonding of the chromium patterns during etching of the sacrificial layer (Fig. 3(d)-(f)). Hence, we experimentally confirmed that 10:1 was the optimal ratio to maximize the stability of the chromium pattern (Fig. 3(c),(f)).

After the curing process, the silicon wafer with PDMS slab was immersed in a $1 \mathrm{M} \mathrm{FeCl}_{3}$ solution to etch the nickel sacrificial layer. The total etching time varied depending on the plasma treatment time (Figure 4(a)). It took over $168 \mathrm{~h}$ for a sample size of $1 \mathrm{~cm}^{2}$, and a plasma treatment time of 1 $\min 30 \mathrm{~s}$. Incomplete etching can induce mechanical failure, when the PDMS is separated from the silicon wafer. Therefore, sufficient time was spent to completely remove the sacrificial $\mathrm{Ni}$ layer, and the progress of the etching process was monitored using the color of the substrate (Fig. 4(b)). Because the special gravity of PDMS is lower than that of the etching solution, the etched part of the PDMS slab experiences an upward buoyant force, causing mechanical stress in the chromium pattern. To prevent this, we placed PDMS on the underside of the substrate (Fig. 4(c),(d)).

\section{Results and Discussion}

We have demonstrated the lithographic capability of the elastomeric photomask. The previous research introduced a lithography technique using an elastomeric photomask [2]. This technique provided a non-planar and high-resolution patterning capability without complex lithography equipment. Photolithography using an elastomeric photomask is similar to conventional contact printing. First, a photoresist (Dow (a)

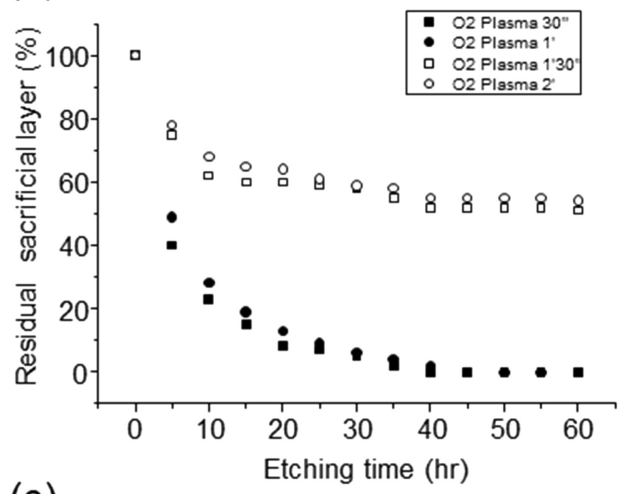

(c)

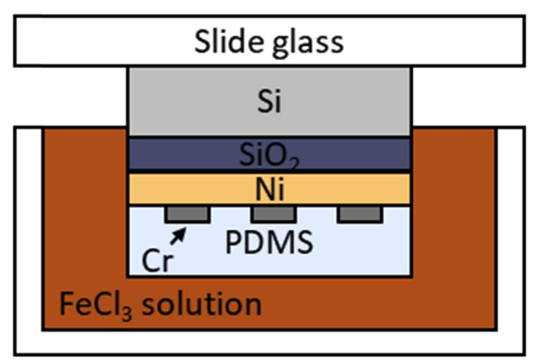

(b)
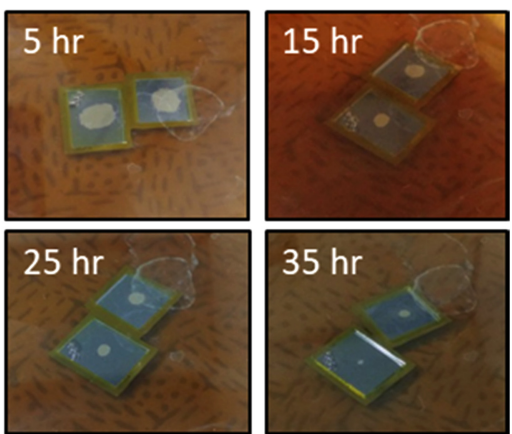

$35 \mathrm{hr}$

(d)

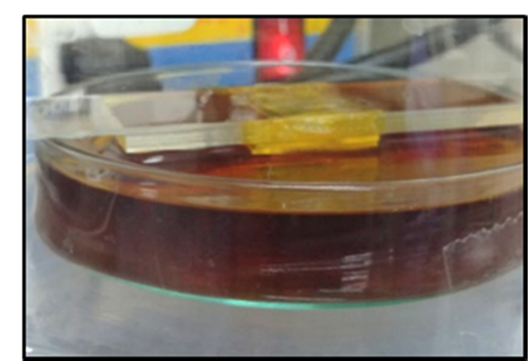

Fig. 4. (a) The ratio of residual Ni sacrificial layer depending on etching time with various $\mathrm{O}_{2}$ plasma times. (b) Pictures of an etched nickel sacrificial layer on a silicon substrate over etching time with $\mathrm{O}_{2}$ plasma time of 1 min. (c) Schematic illustration of Ni sacrificial layer etching process. (d) A picture of Ni sacrificial layer etching process as depicted in (c). 
(a)

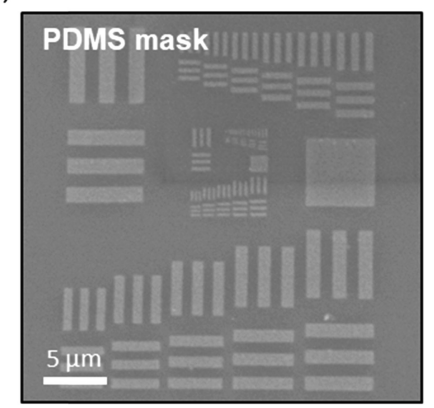

(b)

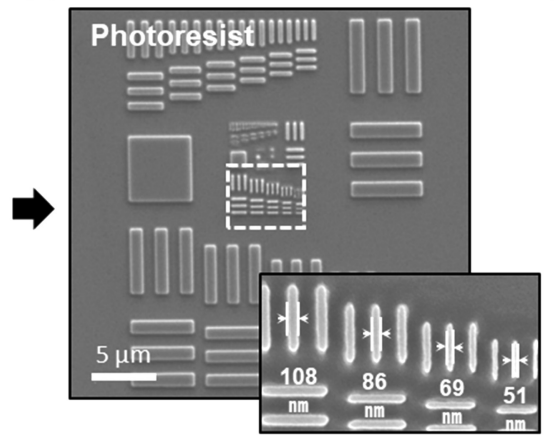

(c)

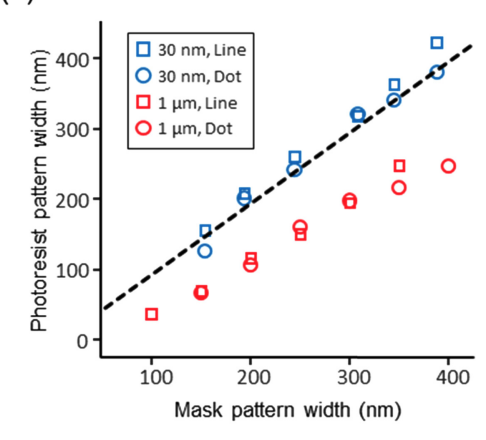

Fig. 5. Scanning electron microscopy images of (a) PDMS mask and (b) corresponding photoresist patterns with an inset image showing an enlarged view. (c) The width of a photoresist feature corresponding to the photomask pattern width for various thicknesses of the photoresist layer.

Electronic Materials, MEGAPOSIT SPR510A) was spincoated onto the substrate. The photoresist could be diluted with the proper solvent to obtain the desired photoresist layer thickness. An elastomeric photomask was brought into contact with the photoresist surface using a conventional contact mask aligner (MIDAS SYSTEM, MDA-400S). The substrate was then exposed to UV light from a mercury-vapor lamp, followed by development with a tetramethylammonium hydroxide solution (2.38 wt.\%, nepes, CPD-BD).

To evaluate the lithography performance of an elastomeric photomask, we used a standard 1951 USAF resolution test chart as a mask pattern. The test patterns are composed of 36 elements of line patterns ranging from $17 \mathrm{~nm}$ to $977 \mathrm{~nm}$ in width. We fabricated an elastomeric photomask of the resolution test patterns using our protocol (Figure 5(a)). Then, the mask pattern was transferred onto a $1 \mu \mathrm{m}$ thick photoresist layer. Fig. 5(b) shows the resulting photoresist features corresponding to the mask patterns shown in Fig. 5(a). The dimensions of the photoresist pattern was slightly smaller than that of chromium pattern, perhaps due to overdevelopment. The smallest feature was $51 \mathrm{~nm}$ in width, resulting from both the resolving power of the gapless contact of the elastomeric photomask and the overdevelopment of the photoresist due to the thick photoresist layer.

To investigate the effect of the thickness of the photoresist layer on the resolution capability, we patterned a dot and line pattern on the silicon substrate with two different thicknesses $(1 \mu \mathrm{m}$ and $30 \mathrm{~nm}$ ) of photoresist. To coat the $30 \mathrm{~nm}$-thick photoresist layer, we used MicroChem S1805 diluted with propylene glycol methyl ether acetate in 1:9 volume ratio and spincoated the diluted photoresist at $3000 \mathrm{rpm}$ for $30 \mathrm{~s}$. Fig. 5(c) shows the width of the photoresist features based on the width of the mask pattern and the thickness of the photoresist. The thick and thin photoresist layers exhibited distinct trends in feature size. In the 30 -nm thick photoresist layer, the width of the photoresist pattern was consistent with an elastomeric photomask, while shrinkage of the photoresist pattern was observed in the $1 \mu \mathrm{m}$-thick photoresist layer. The feature size discrepancy in the thicker layer could be due to diffraction inside the thick photoresist layer, leading to overdevelopment in the vicinity of a pattern.

The excellent resolution of the elastomeric photomask can be attributed to the flexibility of the mask plate, which enables gapless contact with the substrate. In contact printing, the resolving capability is limited by diffraction through the pathway of the incident light, and is given by the following equation:

$$
2 R=3\left[\lambda\left(s+\frac{1}{2} z\right)\right]^{\frac{1}{2}},
$$

where $2 R$ is the grating period, $\lambda$ is the wavelength of the incident light, $s$ is the distance between the mask and the photoresist surface, and $z$ is the thickness of the photoresist layer. In conventional contact printing with a glass photomask, a gap between the mask and the photoresist surface is inevitable because the photomask and the substrate surfaces lack flatness [20], which limits fine patterning below the micrometer scale. In contrast, an elastomeric photomask leaves no gap $(s=0)$ between the mask and the photoresist surface because the flexible mask plate conformally deforms to 
the non-planar profile of the photoresist surface. Conformal contact of the elastomeric photomask prevents diffraction through the gap and enables nanoscale resolving power without any focusing element or short-wavelength light. With cost-efficient equipment, this technique could be effectively applied to nanostructure applications [2,21-23].

\section{Conclusion}

We introduced a reliable protocol to fabricate a PDMS photomask with nanosized chromium mask patterns, and investigated the lithographic performance when contact printing using an elastomeric photomask. By combining the conventional nanofabrication process with a transfer method using a sacrificial layer, our fabrication protocol was able to define a designed metal pattern, and produce a highly stable chromium mask pattern on an elastomeric mask plate. We verified the distinct advantages of the elastomeric photomask by testing its patterning resolution, which achieved a smallest feature size of $51 \mathrm{~nm}$ wide using simple conventional contact printing. We expect that the well-established fabrication process with the elastomeric photomask in this study will permit routine nanolithography processing with both high resolution and low cost.

\section{Acknowledgement}

Sangyoon Paik and Gwangmook Kim contributed equally to this work. This work was supported by the National Research Foundation of Korea (NRF) grant funded by the Korean government (NRF-2018M3D1A1058793, 2015R1A5A1037 668, 2020R1A2C2102150).

\section{REFERENCES}

1. A.K.-K. Wong, Resolution Enhancement Techniques in Optical Lithography. SPIE PRESS, pp.13-18, Bellingham, Washington USA (2001).

2. S. Paik, G. Kim, S. Chang, S. Lee, D. Jin, K.-Y. Jeong, I. S. Lee, J. Lee, H. Moon, J. Lee, K. Chang, S. S. Choi, J. Moon, S. Jung, S. Kang, W. Lee, H.-J. Choi, H. Choi, H. J. Kim, J.H. Lee, J. Cheon, M. Kim, J. Myoung, H.-G. Park, and W. Shim, Nat. commun. 11, 805 (2020).
3. M. M. Alkaisi, R. J. Blaikie, and S. J. McNab, Adv. Mater. 13, 877 (2001).

4. K. J. Lee, K. A. Fosser, and R. G. Nuzzo, Adv. Funct. Mater. 15, 557 (2005).

5. J. G. Kim, N. Takama, B. J. Kim, and H. Fujita, J. Micromech. Microeng. 19, 055017 (2009).

6. H. J. Park, J. H. Yoon, K. G. Lee, and B. G. Choi, Nano Converg. 6, 9 (2019).

7. C. Parameswaran and D. Gupta, Nano Converg. 6, 28 (2019).

8. W. S. Lee, S. Jeon, and S. J. Oh, Nano Converg. 6, 10 (2019).

9. Y. M. Park, S. Y. Lim, S. W. Jeong, Y. Song, N. H. Bae, S. B. Hong, B. G. Choi, S. J. Lee, and K. G. Lee, Nano Converg. 5, 15 (2018).

10. D. Y. Kim, M. -J. Kim, G. Sung, and J. -Y. Sun, Nano Converg. 6, 21 (2019).

11. D. Kim, T. Lee, and D. Choi, Korean J. Met. Mater. 57, 795 (2019).

12. B. Sun, X. Huang, and Z. Li, Met. Mater. Int. 26, 501 (2020).

13. K. S. Ryu, X. Wang, K. Shaikh, and C. Liu, J. Microelectromech. S. 13, 568 (2004).

14. W. Chen, R. H. W. Lam, and J. Fu, Lab Chip 12, 391 (2012).

15. J. Lee, W. Shim, E. Lee, J.-S. Noh, and W. Lee, Angew. Chem. Int. Ed. 50, 5301 (2011).

16. N. J. Douville, Z. Li, S. Takayama, and M. D. Thouless, Soft Matter. 7, 6493 (2011).

17. J. W. Hutchinson, Stresses and Failure Modes in Thin Films and Multilayers. Notes for a Dcamm Course. Technical University of Denmark, pp.4-12, Lyngby, Denmark (1996).

18. J.-Y. Sun, N. Lu, J. Yoon, K.-H. Oh, Z. Suo, and J. J. Vlassak, J. Mater. Res. 24, 3338 (2009).

19. I. D. Johnston, D. K. McCluskey, C. K. L. Tan, and M. C. Tracey, J. Micromech. Microeng. 7, 035017 (2014).

20. L. F. Thompson and M. J. Bowden, The Lithography Process: The Physic in INTRODUCTION TO MICROLITHOGRAPHY. American Chemical Society, p.19, Washington, D. C., USA (1983).

21. T. Lee, J. Jang, H. Jeong, and J. Rho, Nano Converg. 5, 1 (2018).

22. T. Y. Jeon, D. J. Kim, S. -G. Park, S. -H. Kim, and D.-H. Kim, Nano Converg. 3, 18 (2016).

23. T. W. Park, M. Byun, H. Jung, and W. I. Park, Korean J. Met. Mater. 58, 26 (2020). 